\title{
SUSPENDED SEDIMENT DUE TO WAVE ACTION
}

Masash1 Hom-ma and Kiyosh1 Horikawa

Department of Civil Engineering, University of Tokyo Tokyo, Japan

\section{INTRODUCTION}

The sediment movement in the littoral zone occurs in the form of bed load (material rolled and pushed along the bottom by shear stress) and suspended load (material maintained in suspension by action of waves and currents). The present paper is concerned with studies through both the field and laboratory investigations intended to determine the concentration patterns of suspended sediment due to action of surface waves. The distribution patterns of suspended sediment sampled at an open coast were highly interesting in relation to functional and structural design of the cooling water intake system for the atomic power plant now being under construction at the Pacific Ocean coast of Toka1, Ibarag1 Prefecture, Japan.

As far as the authors are concerned, the clear relationship between hydraulic conditions, such as helght and period of waves, water depth, water temperature, etc., and sediment characteristics has not yet been estabilished, although a number of researches have been conducted in the laboratory and in the field. Main of them are as follows:

In Japan: (1) Field observations at Tomakomal coast conducted by the staff of Hokkaido University (Refs. $1,2,3,4,5,6$ and 7),

(2) Experimental study at Kyushu University (Refs. 8 and 9),

(3) Field observations at Katase and Kamakura beaches, Kanagawa Prefecture (Refs. 10, II and 12), and at Toka1 coast, Ibarag1 Prefecture (Refs. 13, 14 and 15), both conducted by the staff of the Coastal Engineering Laboratory, University of Tokyo, and

(4) Field observations at Nilgata coast, Nilgata Prefecture (Refs. 16 and 17). 


\section{SUSPENDED SEDIMENT DUE TO WAVE ACTION}

In the United States: Field observations (Ref. 18) and laboratory investigations (Refs. 19 and 20) at the Beach Erosion Board, Corps of Engineers, U. S. Army.

The present investigation consists of the field studies at Tokal and Nilgata coasts and the laboratory studies at the Coastal EngIneering Laboratory, University of Tokyo. In the field, two types of sampling device have been used, one consisting of instantaneous sampling of sediment suspended in water and the other of cumulative sampling by use of a bamboo staff which is held upright in various depths of water for a period of several days. The latter type was originalIy developed by the researchers at Hokkaido University. In the laboratory studies both syphon tubes and bamboo models were used in a two dimensional wave flume. In parallel to these empirical procedures, a theoretical consideration has been performed on the basis of several assumptions in order to analyze the results of fleld and laboratory investigations.

\section{THEORETICAL CONSIDERATION}

According to visual observations through the glassed wall of the wave flume, sand particles spread over the bottom are moved back and forth by the osciliatory motion of water and gradually form sand ripples having a particular dimension corresponding to the types of waves. The turbulence of the fluid is originally restricted to a very thin layer in the vicinity of the flat bottom, but is greatly magnified with formation of sand ripples. The vortices developed in the troughs of sand ripples travel upward, imparting a strong turbulence to almost the entire depth from bottom to surface and carrying with them concentration of bottom sediment. It is therefore realized that sand ripples may have an important effect on the mechanics of suspended sediment subjected to wave action.

We will now assume a two-dimensional turbulent fluid, and proceed to formulate a theoretical distribution pattern of suspended sediment. Taking the horizontal coordinate, $x$, in the direction of wave propagation and the vertical coordinate, $z$, upward from the still water level, the equation of sediment concentration is given by the well-known expression: 


$$
w_{0} \frac{\partial m}{\partial z}+\frac{\partial}{\partial z}\left(\varepsilon \frac{\partial m}{\partial z}\right)=0
$$

where wo is the fall velocity of a sand particle, $m$ the concentration of suspended sediment, and $\varepsilon$ a coefficient of eddy viscosity. In analogy to the classical theory of turbulence, we will assume the following relationship for the value of $\varepsilon$,

$$
\varepsilon=\beta b^{2}\left|\frac{\partial u}{\partial z}\right|
$$

In which $b$ and $u$ are the minor radius of an orbit and the horizontal velocity component of water particle, respectively, in the presence of oscillatory motion by waves; and $\beta$ is considered to be practically constant. As a first approximation, $\eta$, vertical displacement of water surface above stili water level, $b$ and $u$ may be expressed as:

$$
\begin{aligned}
& \eta=\frac{H}{2} \sin \left(k x-\frac{2 \pi}{T} t\right), \\
& b=\frac{H}{2} \frac{\sinh k(z+d)}{\operatorname{sinhkd},} \\
& u=\frac{\pi H}{T} \frac{\cosh k(z+d)}{\operatorname{sinhkd} \sin \left(k x-\frac{2 \pi}{T} t\right),} \\
& k=\frac{2 \pi}{L},
\end{aligned}
$$

where $H . I$ and $T$ are wave height, wave length and wave period, respectively, at the water depth d. Now, we will further assume the following expression for $\mathrm{m}$ :

$$
m=\bar{m}(z) \cdot\left\{1+\xi \sin 2\left(k x-\frac{2 \pi}{T} t\right)\right\}
$$

Introducing Eqs. (2), (4), (5) and (7) into Eq. (1) and taking a time average over a cycle of wave period $T$, we have the differential equation: 


$$
w_{0} \frac{d \bar{m}}{d z}+\frac{d}{d z}\left(\frac{\beta H^{3} k}{3 T} \frac{\sinh ^{3} k(z+d)}{\sinh ^{3} k d} \frac{d \bar{m}}{d z}\right)=0
$$

Integration of $\mathrm{Bq}$. (8) by considering the boundary condition at the surface and the relationship $\bar{m}=\bar{m}_{a}$ at $z=-a$, reduces to

$$
\frac{\bar{m}}{\bar{m}_{2}}=\exp \left[-\frac{3 w_{0} T}{\beta H^{3} k} \sinh ^{3} k d \int_{-a}^{z} \frac{d z}{\sinh ^{3} k(z+d)}\right] \text {. }
$$

Rewriting $\mathrm{Eq}_{\mathrm{q}}$. (9), we arrive at the final expression:

$$
\begin{aligned}
& \frac{\bar{m}}{\bar{m}_{a}}=\exp \left[-\alpha \frac{w_{0}}{c}\left(\frac{L}{H}\right)^{3}\left(\frac{d}{L}\right) \sinh ^{3} k d \cdot f(n, k)\right], \\
& f(n, k)=\int_{n_{a}}^{n}(\operatorname{sinhknd})^{-3} d n=\frac{1}{2 d k}\left(\left\{\frac{\cosh k n_{a} d}{\sinh ^{2} k_{2} d}\right.\right. \\
& \left.\left.-\frac{\cosh k n d}{\sinh h^{2} \text { knd }}\right\}+\log \left|\frac{\tanh \frac{\ln _{a} d}{2}}{\tanh \frac{\operatorname{kn} \alpha}{2}}\right|\right) \text {, } \\
& \alpha=\frac{3}{\beta}, \quad c=\frac{L}{T}, \quad n=\frac{d+z}{d}, \quad n_{2}=\frac{d-\alpha}{d} .
\end{aligned}
$$

If the values of $\alpha$ and $\bar{m}_{a}$ at $n_{a}$ are determined experimentally, the concentration of suspended sediment at an arbitrary level can be calculated by using Eq. (10) for variable conditions of $\mathrm{H}, \mathrm{I}, \mathrm{T}, \mathrm{d}$ and wo. The term wo is closely related to sediment characteristics, namely grain diameter, and also the temperature of fluid.

\section{FIELD OBSERVATIONS}

In the field observations conducted at the beaches of Katase, Kamakura and Tokai, it was impossible to derive a generalized pattern of suspended sediment in terms of absolute quantity. It was due mainly to fallure of adequate measurement of waves during the period of observation, although abundant data on the amount of suspended sediment were made available by bamboo samplers. However, the later phase of investi- 


\section{COASTAL ENGINEERING}

gation included the wave measurement done with special care simultaneously with the sediment sampling operation. A pressure-type self-contained wave gauge was installed on the sea bottom at the depth of about $10 \mathrm{~m}$ below the mean sea level, about $500 \mathrm{~m}$ offshore, in the field observation at the Tokal coast.

A horizontal-type instantaneous sampler, capacity being $1.5 \mathrm{l}$, was devised for direct measurement of vertical distribution of sediment concentration, but it proved unfeasible due mainly to the difficulty of operation under rough sea conditions.

A cumulative bamboo sampler enables to determine a cumulative load of suspended sediment, but there exist several uncertainties as to the function of this type of sampler. They are: (1) What is the relationship between the cumulative amount of sediment collected by this sampler and the actual concentration of sediment? (2) What governs the effectiveness of sediment trapping mechanism by this sampler? In spite of such difficulties the authors have found so far this sampling device most preferable for use in the field observations. In order to clarify the said uncertainties of a bamboo sampler, a comparison test was necessary by using model samplers in a laboratory flume.

\section{LABORATORY INVESTIGATIONS}

The wave flume used for the comparison test is two-dimensional, $24 \mathrm{~m}$ long, $0.8 \mathrm{~m}$ deep and $1.0 \mathrm{~m}$ wide. Two types of sand, $175 \mu$ and $100 \mu$ in medium diameter, $M d$, were used. The suspended sediment was sampled, instantaneously, with a simple device of syphon tube, $6 \mathrm{~mm}$ I.D., which was equipped with a T-shaped suction mouth, and, cumulatively, with a plastic cylinder, $2.5 \mathrm{~cm}$ I.D. and $9 \mathrm{~cm}$ high, with a pair of openings of $1 \mathrm{~cm}$ diameter on the opposite sides. (FIgs. 1, 2 and 3)

The temperature of water was carefully measured, since it may affect fluid viscosity and hence the suspended concentration, as suggested by Fairchild (Ref. 20), al though the temperature effect seemed insignificant in the range of our experiment. The bottom level was difficult to define because of the presence of sand ripples. For the sake of convenience, we have used the original smooth bottom as the reference level for each run of experiment. 
SUSPENDED SEDIMENT DUE TO WAVE ACTION

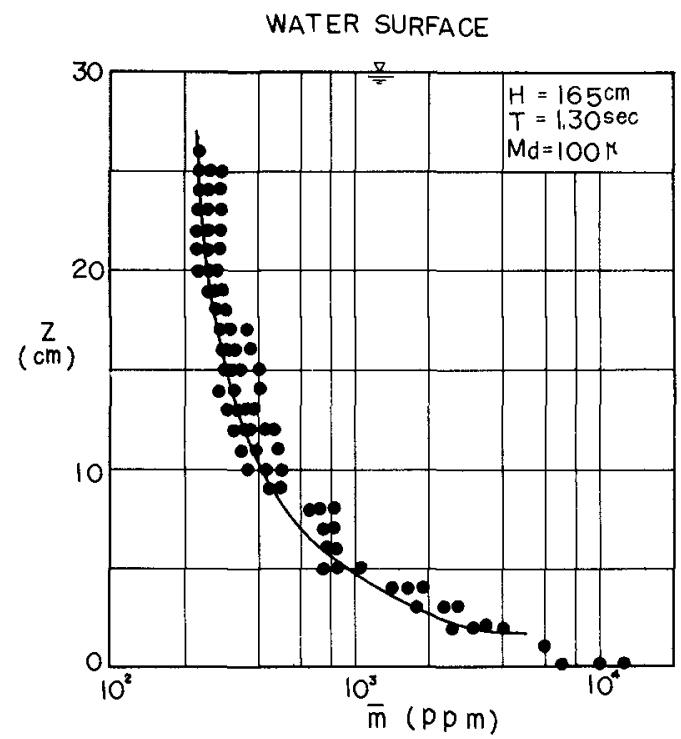

Figure 1. Vertical distribution curve of suspended sediment concentration (Coastal Engineering Laboratory, University of Tokyo).

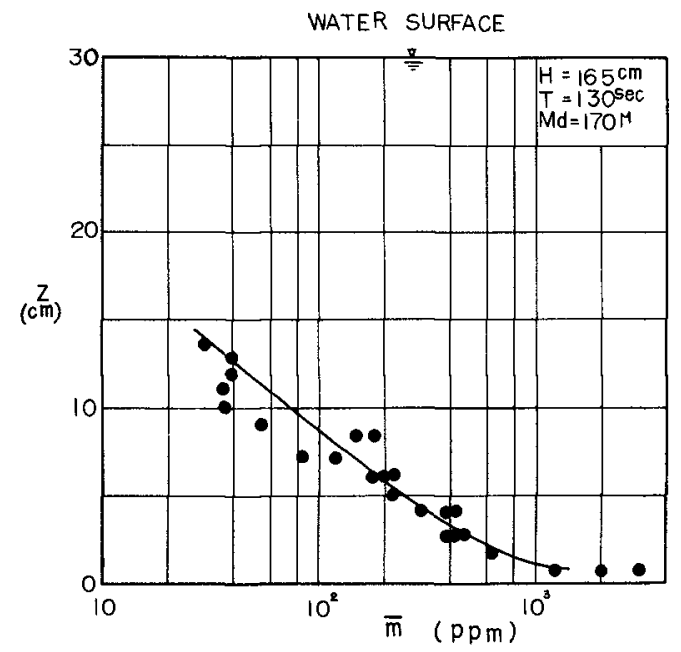

Figure 2. Vertical distribution curve of suspended sediment concentration (Coastal Engineering Laboratory, University of Tokyo). 


\section{COASTAL ENGINEERING}

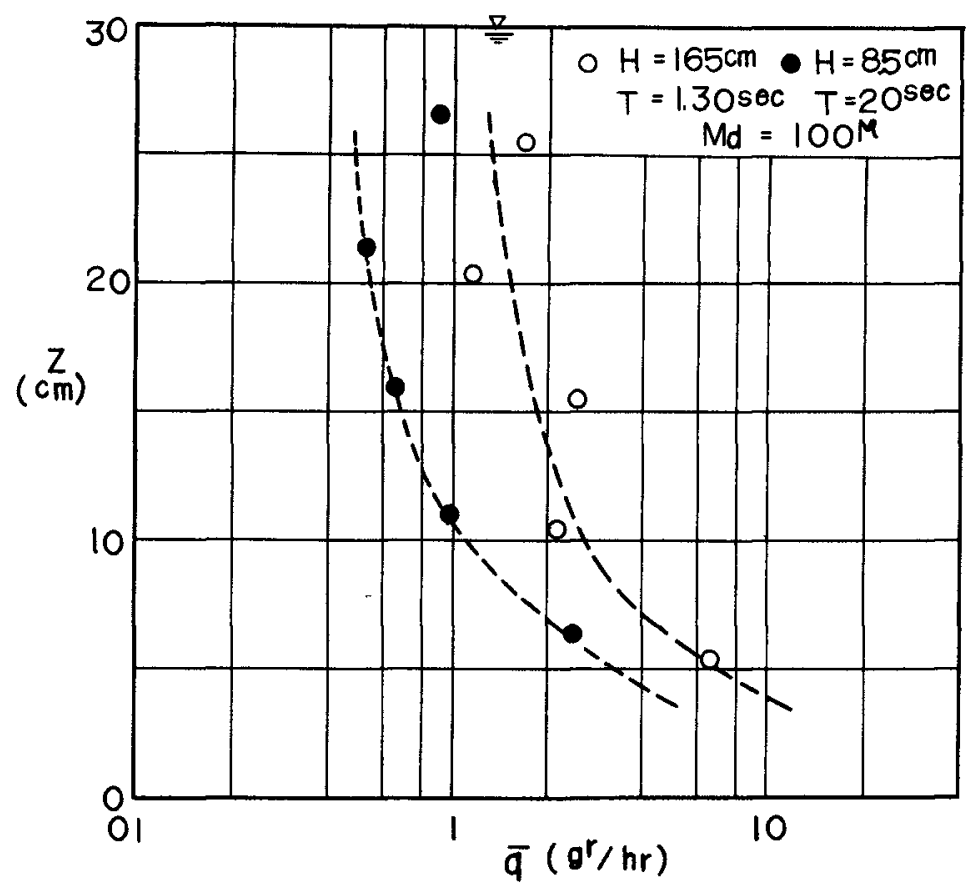

Figure 3. Vertical distribution curve of suspended sediment, cumulative amount by a bamboo model.

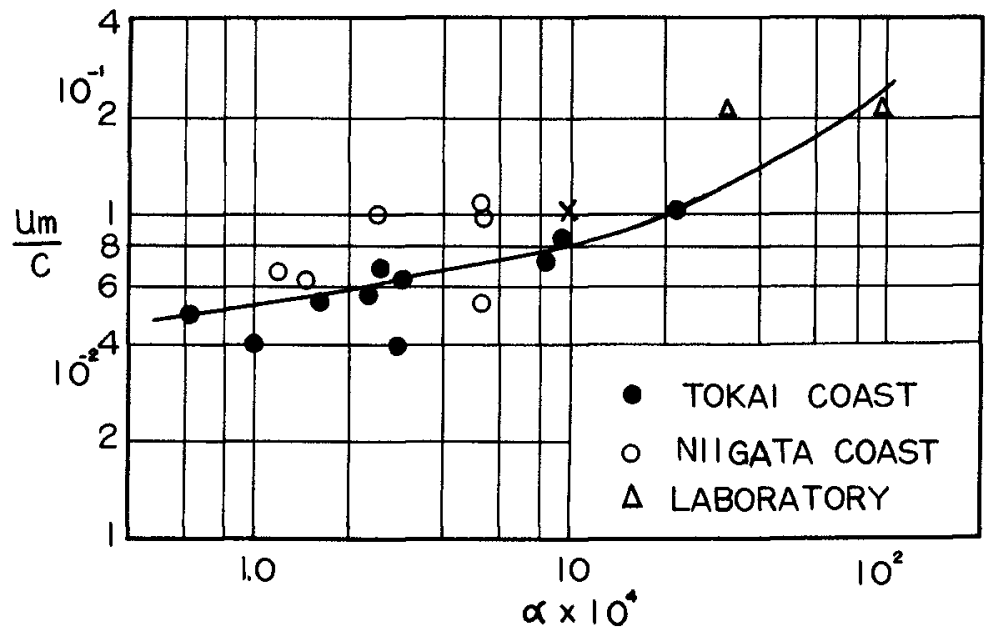

Figure 4. Relationship between $\bar{\alpha}$ and $u_{m} / c$. 
SUSPENDED SEDIMENT DUE TO WAVE ACTION

Table 1. Calculated values of $\alpha$.

(I) Laboratory (syphon tube, instantaneous)

\begin{tabular}{|c|c|c|c|c|c|c|}
\hline $\begin{array}{l}\text { Wave } \\
\text { Height } \\
\mathrm{H}(\mathrm{cm})\end{array}$ & $\begin{array}{l}\text { Wave } \\
\text { Period } \\
\text { T(sec) }\end{array}$ & $\begin{array}{l}\text { Water } \\
\text { Depth } \\
d(d m)\end{array}$ & $\mathrm{n}$ & $\alpha$ & $\bar{\alpha}$ & $\begin{array}{l}M_{d} \\
(\mu)\end{array}$ \\
\hline 16.5 & 1.30 & 30 & $\begin{array}{l}0.2 \\
0.3 \\
0.4\end{array}$ & $\begin{array}{l}1.9 \times 10^{3} \\
2.8 \times 10^{-3} \\
4.9 \times 10^{-3}\end{array}$ & $3.2 \times 10^{-3}$ & 170 \\
\hline 16.5 & 1.30 & 30 & $\begin{array}{l}0.2 \\
0.3 \\
0.5 \\
1.0\end{array}$ & $\begin{array}{l}7.6 \times 10^{-3} \\
9.2 \times 10^{-3} \\
11.1 \times 10^{-3} \\
10.3 \times 10^{-3}\end{array}$ & $9.5 \times 10^{-3}$ & 100 \\
\hline
\end{tabular}

(2) Field (bamboo sampler, cumulative, Toka1)

\begin{tabular}{|c|c|c|c|c|c|}
\hline $\begin{array}{l}\text { Wave } \\
\text { Height } \\
\overline{\mathrm{H}}_{1 / 3} \\
\text { (m) } \\
\end{array}$ & $\begin{array}{l}\text { Wave } \\
\text { Period } \\
\mathrm{T}_{1 / 3} \\
(\mathrm{sec}) \\
\end{array}$ & $\begin{array}{c}\text { Water } \\
\text { Depth } \\
\mathrm{d} \\
(\mathrm{m})\end{array}$ & $\mathrm{n}$ & $\alpha$ & $\bar{\alpha}$ \\
\hline 1.09 & 6.2 & 4.8 & $\begin{array}{l}0.21 \\
0.28 \\
0.30 \\
0.43 \\
0.58 \\
0.73\end{array}$ & $\begin{array}{l}1.3 \times 10^{3} \\
1.5 \times 10^{-3} \\
1.6 \times 10^{3} \\
1.7 \times 10^{-3} \\
1.7 \times 10^{-3} \\
1.9 \times 10^{-3}\end{array}$ & $1.6 \times 10^{3}$ \\
\hline 1.06 & 6.2 & 7.8 & $\begin{array}{l}0.22 \\
0.31 \\
0.41 \\
0.52\end{array}$ & $\begin{array}{l}2.1 \times 10^{-4} \\
2.1 \times 10^{-4} \\
2.2 \times 10^{-4} \\
2.6 \times 10^{-4}\end{array}$ & $2.3 \times 10^{-4}$ \\
\hline
\end{tabular}




\section{ANALYSIS OF DATA}

The concentration of suspended sediment tends to increase distinctly from water surface to bottom. The increase is especially pronounced near the bottom, producing a distribution curve with the shape of " ( ", as previously pointed out by Fukushima et al (Ref. 1). One of the most realistic explanation of this fact may be that the sand particles are picked up and maintained in suspension by the vortices formed in the ripple troughs and separated upward following the propagation of surface waves. The detailed mechamism of the above processes being still unknown, the existence of a fully developed turbulence has been assumed in the theoretical treatment as mentioned in the previous section. The vertical distribution curve of sediment amount trapped by a bamboo sampler is completely simflar to that of the actual sediment concentration. In the following analysis the value at $n=0.1$ will be consistently taken as the reference in view of the fact that almost all the data include the measured values of $\overline{\mathbf{m}}$ or $\bar{q}$ at the elevation of $10 \%$ of the total water depth above the bottom.

CALCULATED VALUE OF $\alpha$

The value of $\alpha$ for each level is calculated, as listed in part in Table I, by using the theoretical relationship. Reviewing the above result, it is recognized that $\alpha$ seems to vary slightly with the value of $\mathrm{n}$ depending upon hydraulic and sedimentary conditions. For the sake of simplicity, we will use the mean value, $\alpha$, in the following discussions.

Figure 4 shows the variation of $\bar{\alpha}$ in relation to the variation of nondimensional term of $\frac{u_{m}}{t}$, where $u_{m}$ is a maximum orbital velocity in the vicinity of the bottom and $c$ the celerity of waves. The data obtained both in the field (Tokal and Nilgata coasts) and in the laboratory have a consistent tendency in the graph in spite of scattering of data.

$\bar{m}_{\mathrm{a}}$ AND $\bar{q}_{a}$

In order to determine quantitatively the concentration pattern of suspended sediments, we must know the value of $\bar{m}_{a}$. Fig. 5 shows that $\bar{m}_{a}$ is closely related with the value of $u_{m}$. But the field data (Tokai coast) deviate from and run parallel to the laboratory data which include that of Fairchild of the Beach Erosion Board. A possible reason for this pronounced discrepancy can be found in the difference of 
SUSPENDED SEDIMENT DUE TO WAVE ACTION

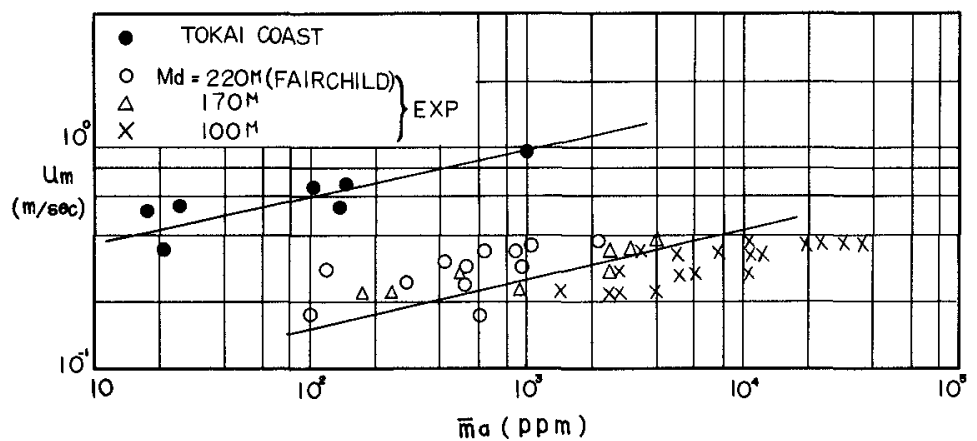

Figure 5. Relationship between $\overline{\mathbf{n}}_{a}$ and $u_{m}$.

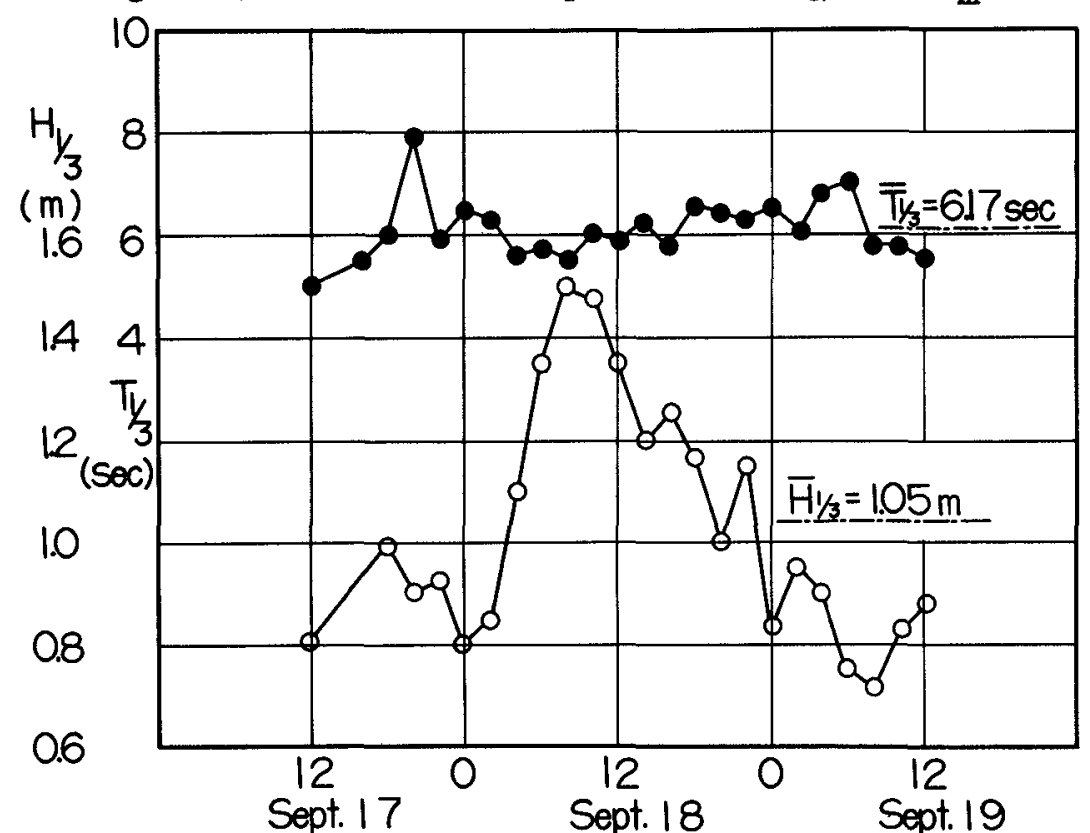

Figure 6. Bxample of wave data.

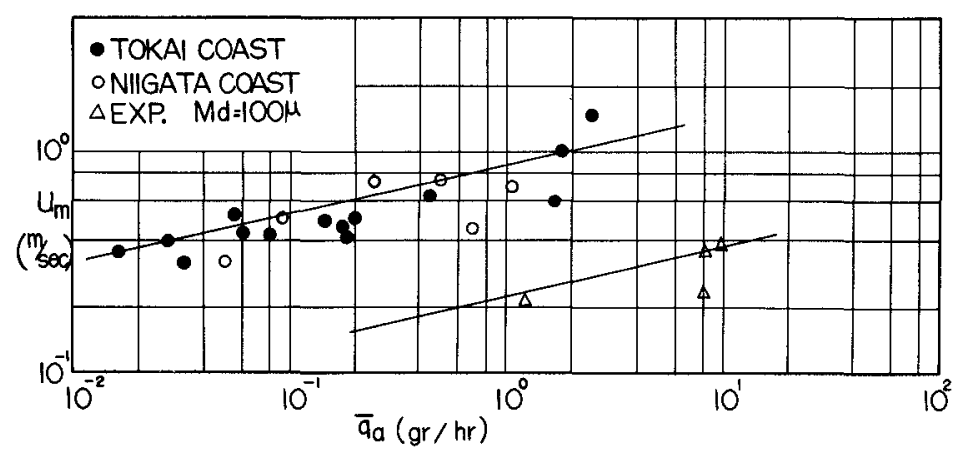

Figure 7. Relationship between $\bar{q}_{a}$ and $u_{m}$. 
data processing. Since the wave in the field would tend to fluctuate as shown in $F 1 g$. 6 , we have used the mean values of significant wave height and period, $\overline{\mathrm{H}} 1 / 3$ and $\overline{\mathrm{T}} 1 / 3$, respectively. On the other hand, the laboratory waves are very simple and their heights and periods are uniquely determined. The solid lines drawn in the figure may be applied to the present problem of fine sediment, although the grain size seems to have some effect on the relationship between $u_{m}$ and $\bar{m}_{a}$.

In the same manner, the relationship between $\bar{q}_{a}$ and $u_{m}$ is given in Fig. 7. The plotting of the se data is completely similar to that in Fig. 5. From the se figures the value of $\bar{q}_{a}$ is correlated with the value of $\bar{m}_{a}$ by using the intermediate parameter $u_{m}$, as shown in Fig. 8. The agreement of the two curves, one from the field and the other from laboratory measurements, is rather surprisingly well. Practically, it is extremely difficult to conduct an instantaneous sampling of suspended sediment under rough sea conditions, while the cumulative sampling with a bamboo sampler is less affected by sea conditions. From such a point of view, the authors believe that the correlation curve presented here may have a practical value in interpreting the data obtained by a bamboo sampler in terms of the equivalent sediment concentration.

\section{COMPARISON OF DATA WITH ANALYTICAL VALUES}

As a result of the analysis which has been described above, a concentration patter of suspended sediment $c$ an be determined after some numerical computation for given conditions of $H, T, d, M_{d}$ and temperature of water. Several examples of comparison between measured data and analytical values are given in Figs. 9 and 10. Generally speaking, the agreement is satisfactory for engineering purposes.

The discussions so far presented have proved useful in determining the adequate height of the mouth of an intake pipe for the cooling water system of the atomic power plant at Tokal, Japan. Analyzing a longterm recording of wave height and perlod at the Tokai coast, the authors could estimate the moderate combination of significant wave characteristics in the rough sea conditions in the water of $8 \mathrm{~m}$ depth where the intake unit will be placed. The wave conditions are as follows. (Table 2)

The median diameter of bottom sediment has been assumed to be $250 \mu$. The value of $\bar{\alpha}$ for such a large value of $u_{m} / c$, as shown in Table 2, cannot be determined accurately from Fig. 4. Hence, the authors 
SUSPENDED SEDIMENT DUE TO WAVE ACTION

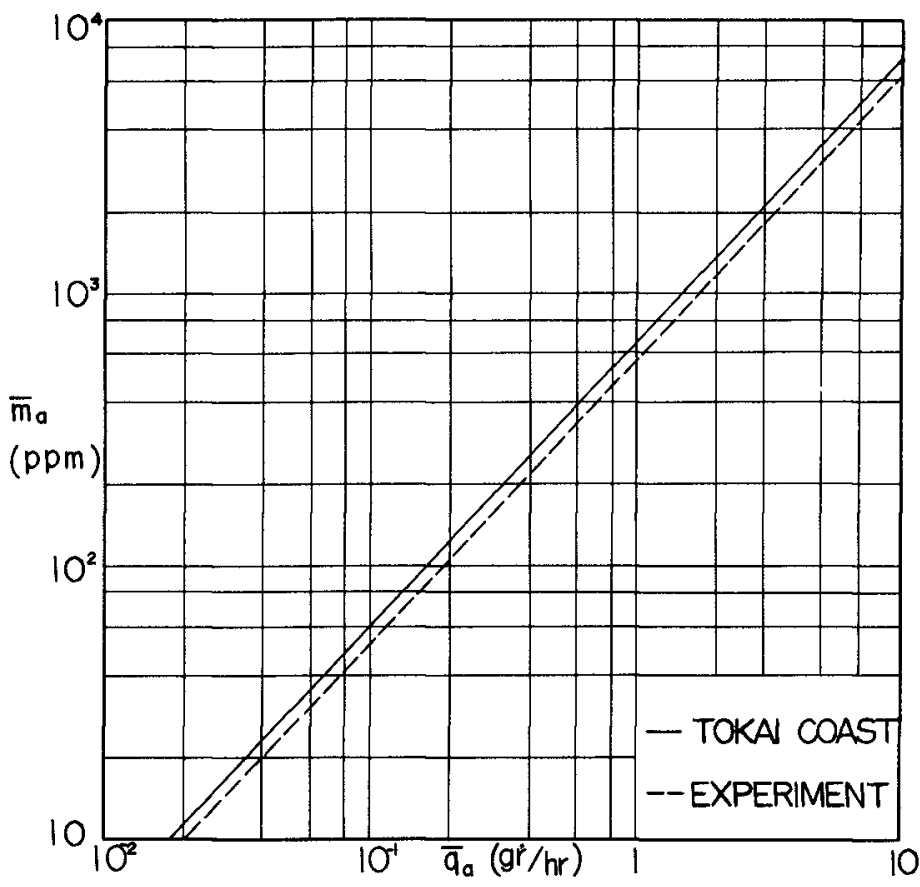

Figure 8. Relationship between $\bar{m}_{a}$ and $\bar{q}_{a}$.

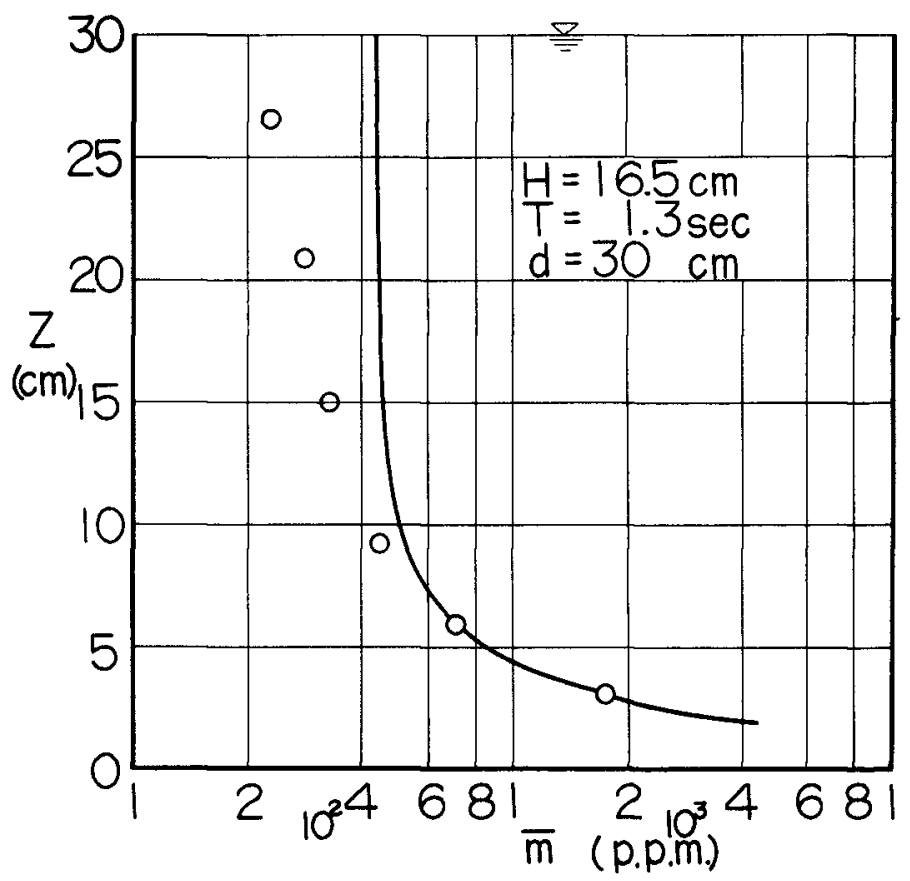

Figure 9. Comparison between analytical curve and measured data (laboratory). 
COASTAL ENGINEERING

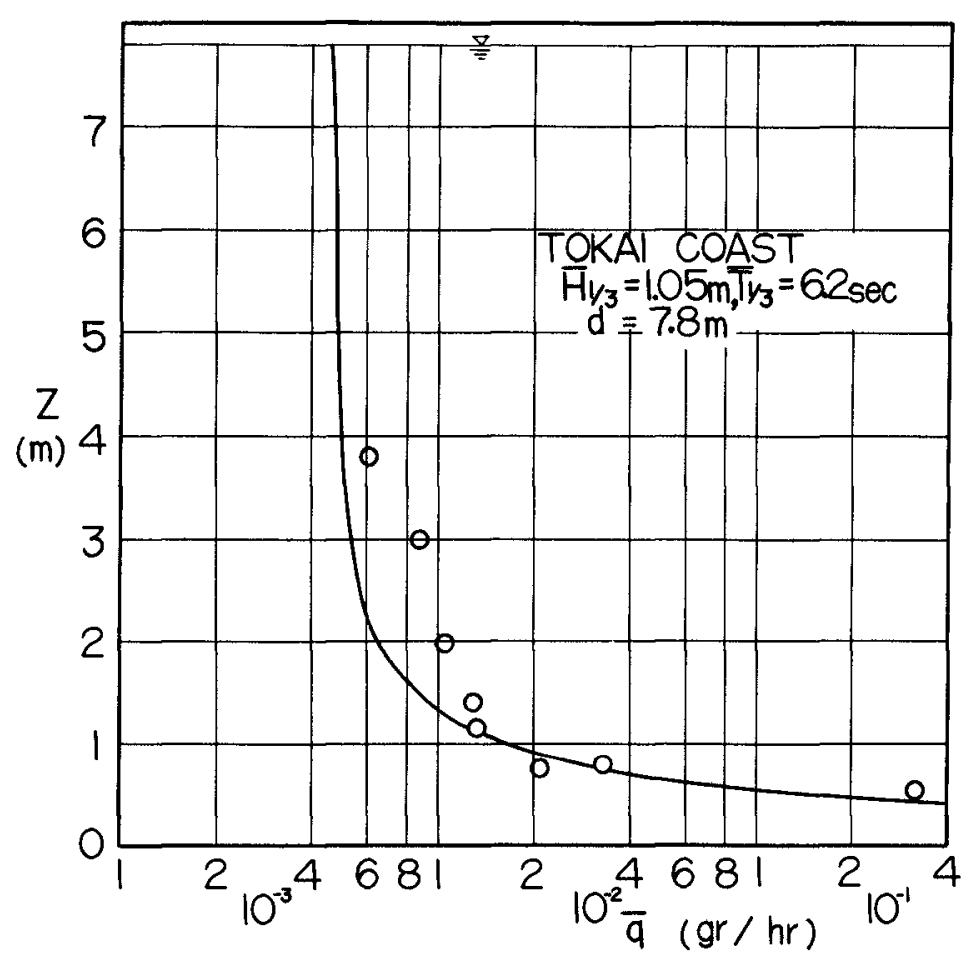

Figure 10. Comparison between analytical curve and measured data (field).

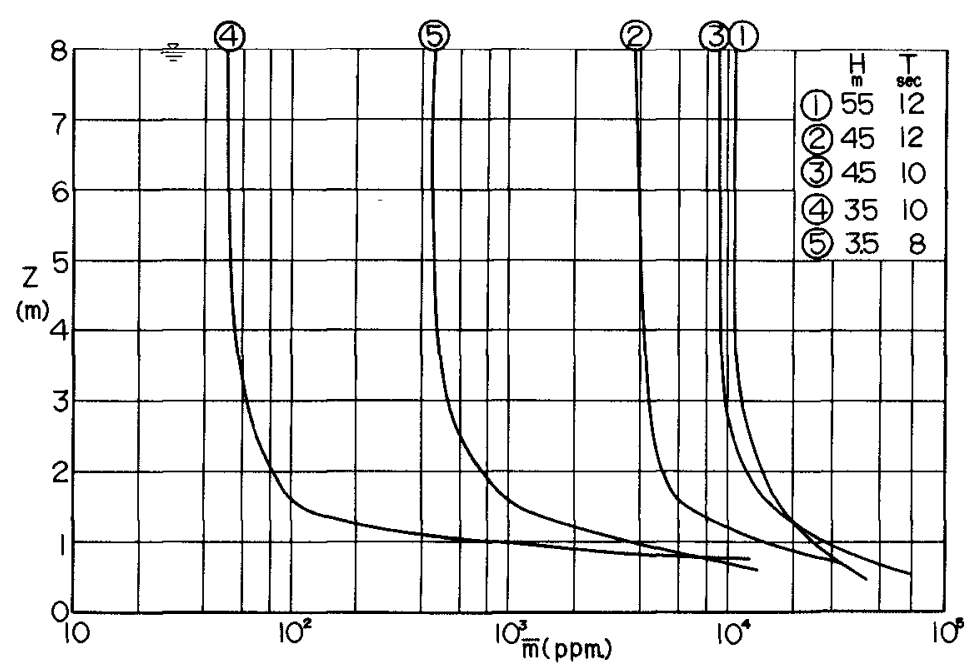

Figure 11. Analytical vertical distribution curves of suspended sediment concentration for variable wave conditions. 
have chosen a unique value of 0.01 for $\bar{\alpha}$ for all conditions considered. The results of computation are shown in Fig. II, from which the following observation may be drawn.

(I) Up to about $2 \mathrm{~m}$ above the bottom the concentration of suspended sediment is considerably high.

(2) Above this level the concentration is rather uniform, and may reach 102 to $10^{4} \mathrm{p} \cdot \mathrm{p} . \mathrm{m}$. depending upon the conditions of waves.

Therefore, it was recommended to set the mouth of the intake pipe beyond $2 \mathrm{~m}$ above the bottom.

Table 2

\begin{tabular}{|c|c|c|}
\hline $\begin{array}{c}\text { Wave Height } \\
\text { in m }\end{array}$ & $\begin{array}{c}\text { Wave Period } \\
\text { in sec }\end{array}$ & $u_{\mathrm{m}} / \mathrm{c}$ \\
\hline 5.5 & 12.0 & 0.263 \\
4.5 & 12.0 & 0.252 \\
4.5 & 10.0 & 0.265 \\
3.5 & 10.0 & 0.206 \\
3.5 & 8.0 & 0.200 \\
\hline
\end{tabular}

SOME RELATED CONSIDERATIONS

In the foregoing sections the phenomena of suspended sediment due to oscillatory wave motion have been treated in a very simplified form. However, the authors realize that the actual phenomena are so complicated that further analysis is necessary in order to complete the treatment. In fact, we have neglected the presence of sand ripples in the analytical procedure, since it is almost impossible at the present moment to treat the problem of a sand ripple in an analytical manner.

\section{SAND RIPPLES}

The authors have conducted a separate study on the behaviors of a sand ripple which will be described briefly in the accounts to follow. It has been repeatedly stressed in the previous sections that the sand ripples have an important role on the occurrence of suspended concentration. It is from recognition of this fact that the authors are interested in the formation and stabilized configuration of sand ripples which appear on the bottom of sediment materials. 


\section{COASTAL ENGINEERING}

Bagnold conducted his original study on this subject by using an oscillating plate in still water with variable size and specific gravity of sediment materials. (Ref. 2l) Manohar did his extensive studies with a similar device at the University of California, introducing valuable conclusions on the behaviors of sand ripples, especially on formation, development and disappearance. (Ref. 22) on the other hand, Scott presented laboratory data on the characteristics of sand ripples, which were formed on the sloping bed due to a surface wave (Ref. 23). While these studies were accomplished in the laboratories, Inman carried out a unique field observation. (Ref.24)

Three questions may be raised as to the significant aspects of a sand ripple.

(1) What conditions may give rise to sand ripples?

(2) What are the stabilized configuration of sand ripples?

(3) What conditions may cause sand ripples to disappear?

The authors will confine themselves mainly to the second question. It is well known that the shape of sand ripoles formed by an oscillatory wave motion is almost symmetrical, different from the asymnetrical ripoles formed by a uni-directional current. Therefore, the shape of the oscillatory sand ripple may well be expressed simply by its rise, $\eta$, and pitch, $\lambda$.

We w1ll consider some elementary factors which may affect the shape of sand ripples, namely:

$u_{m}:$ horizontal component of maximum orbital velocity in the vicinity of bed,

$d_{0}$ : horizontal displacement of water particle in the vicinity of bed,

$\mathrm{Md}_{\mathrm{d}}$ : medium diameter of bed material,

Wo: fall velocity of sediment,

$\nu$ : kinematic viscosity of fluid, and

$\theta$ : temperature of fluid.

As $f\left(w_{0}, M_{d}, \theta\right)=0$, the following relationship may be introduced by dimensional analysis,

$$
\varphi\left(\frac{\eta}{\lambda}, \frac{\lambda}{d_{0}}, \frac{\eta}{d_{0}}, \frac{u_{m} d_{0}}{\nu}, \frac{w_{0} M d}{\nu}\right)=0
$$


in which $\frac{\lambda}{d_{0}}$ and $\frac{\eta}{d_{0}}$ are the relative pitch and relative rise of a sand ripple, respectively, with respect to the horizontal displacement of particle in the vicinity of bed, and $\frac{\eta}{\lambda}$ is the steepness of ripple. other terms are $\frac{u_{m} d}{r}$ and $\frac{w_{0} M d}{v}$, the Reynolds numbers related to the oscillatory motion and the sediment material, respectively.

Prior to an analysis of available data, extensive references have been made to the existing knowledge on the characteristics of wake which is developed behind a circular cylinder in order to utilize an analogy between the walke and formation of a vortex in the ripple trough. (Refs. 25, 26 and 27) In the wake phenomenon the periodical formation and separation of vortices has been noticed and studied experimentally by many authors. The shedding frequency of the wake is usually expressed in terms of the dimensionaless Strouhal number $s=n d / U_{0}$, where $n$ is the shedding frequency (from one side of the cylinder), d the cylinder diameter and $U_{0}$ the free-stream velocity. As a result of experiments, it is known that the strouhal number is a function of the Reynolds number $\frac{U_{o d}}{h}$, which may approach a constant in the range of Large Reynolds number.

Assuming $n=\frac{2 \pi}{T} \quad, d=2 \eta$ and $U_{0}=u_{m}$, the Strouhal number in our case may be expressed as follows:

$$
S=\frac{2 \pi \times 2 \eta}{T U_{m}}=4 \eta \frac{\pi}{T U_{m}}=4 \frac{\eta}{d_{0}} \text {. }
$$

On the other hand, the Reynolds number may be expressed:

$$
R_{e}=\frac{2 u_{m} \eta}{V}
$$

The existence of some definite relationship betThe existence of some definite relationship bet-
ween $\frac{\eta}{d o}$ and $\frac{u m}{h}$ is suggested by the above discus-
sions. sider that the space between the successive ripple crests is occupied by a single vortex formed behind a ripple crest. The size of the vortex must be a function of Reynolds number $\frac{u_{m} \eta}{t}$, similarly to the vortex size of a wake behind a bluff body. Hence, the authors expected the existence of a relationship between $\frac{\eta}{d q}$ and $\frac{\eta}{\lambda}$, and attempted to verify this assumption by using the data of Imman (field), and Scott (I aboratory) and the authors (Iaboratory) as shown in Fig. 12. The result is consistent to a surprising degree, and further intimates that the maximum steepness of a sand ripple seems to be about 0.23 which is approximately equal to $1 / 2$ tan $\phi$, where $\phi$ being the angle of repose of fine sand in water. 


\section{COASTAL ENGINEERING}

In the next step, a relationship among three other terms in Eq. (ii), i.e. $\frac{d_{0}}{\lambda}, \frac{u_{m} d_{0}}{\text { and } w_{0} M d}$ was investigated by using the same data as shown in Fig. 13. From this figure, it may be recognized that the parameter of $\frac{w_{o} M_{d}}{1}$ or $M_{d}$ can be introduced in the relationship betweén $\frac{d o}{\lambda}$ and $\frac{\text { lmde }}{\nu}$ if more data for relatively coarse sand could be plotted in the same figure. The lower limit of $\frac{d_{0}}{\lambda}$ must be 1.0 as previously pointed out by Inman.

According to the above results, the ripple dimensions, $\lambda$ and $\mu$, of fine sand $(300 \mu>M d>100 \mu)$ can be definitely determined for various combinations of wave characteristics, water depth and water temperature in the limited range of the plotted data.

In the above treatment the values of $u_{m}$ and $d_{o}$ were calculated through a first approximation of the oscillatory wave theory, and the value of $v$ was estimated to be $1.00 \times 10^{-2} \mathrm{~cm}^{2} / \mathrm{sec}$.

In the previous sections which have dealt with suspended sediment, both the field and laboratory data were used on the same basis neglecting the scale effect. However, the scale effect must be expected to some extent in view of the following considerations. As pointed out previously, the sand ripples have an $1 \mathrm{~m}-$ portant role on the suspended sediment, but the similarity between prototype and model of ripple dimensions is not reallzed in Figs. 12 and 13, 1.e. the sand ripples are relatively exaggerated in the model.

The authors are inclined to assume that the data by Bagnold and Manohar result from an experimental procedure which may not fully represent the oscillating motion by surface waves. Therefore, they have not been used in our analysis on the characteristic discussion of oscillatory sand ripples. The data of Manohar plotted on the graphs in a similar way to FIgs. 12 and 13 show a fairly large scatter al though they too agree with the upper $11 \mathrm{mits}$ of $\frac{\eta}{\lambda}$ or $\frac{d_{0}}{\lambda}$ versus $\frac{\eta^{2}}{d o}$ or the Reynolds number, respectively At the present moment, the authors feel to share the view of Vincent (Ref. 28) who admits a distinct discrepancy between the motions near the bed produced by an osc1llating bed and by progressive surface wave.

Another point which must be mentioned is the fact that the measured pitch of sand ripples produced by a single train of waves is not unique but varies over a wide range as shown in FIg. 14. Despite scatter, the curve may be considered to represent a normal distribution, hence it may be allowed to take an arithmetic 
SUSPENDED SEDIMENT DUE TO WAVE ACTION

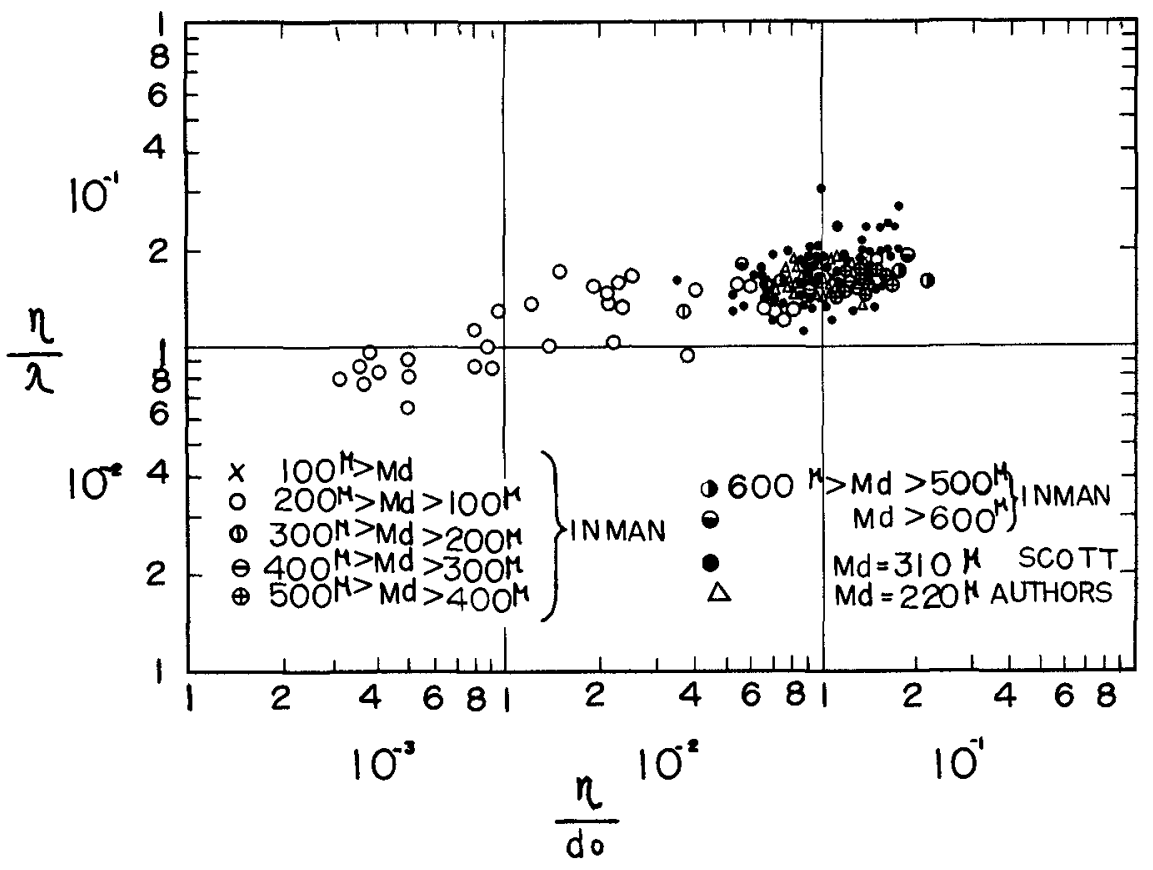
Figure 12. Relationship between $\eta / \lambda$ and $\eta / d$.
(oscillatory sand ripples).

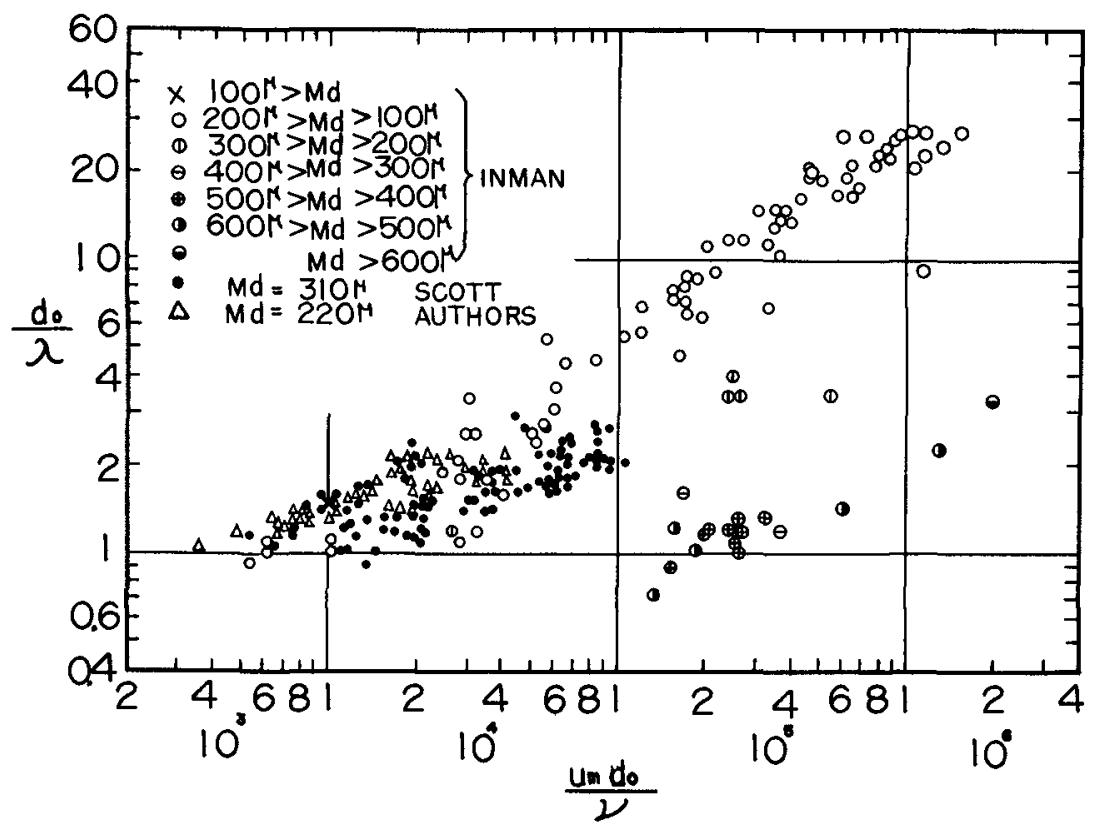

Figure 13. Relationship between $d g / \lambda$ and $U_{m d g} /$ (oscillatory sand ripples). 


\section{COASTAL ENGINEERING}

mean as a representative pitch of the measured samples. The rise also fluctuates to almost the same degree.

VERTICAL DISTRIBUTION OF GRAIN SIZE OF SUSPENDED SED MMENT

In the foregoing treatment the grain size of suspended particles has been assumed for the sake of approximation to be uniform from water surface to bottom. However, as would be expected quite naturally, the grain size tends to decrease gradually upwards owing to the sorting action of turbulence in water. This tendency is clearly shown in Fig. 15, which is based on the data obtained at the Toka1 coast. Beyond a certain level above the bottom the grain size is practically uniform. The data from the Nilgata coast, which give a similar trend, also show that the grain size of suspended sediment at rough sea conditions is larger than that found at calm, even though the bottom sediment is the same. (Fig. 16) Our assumption of a uniform grain diameter for suspended sediment seems therefore substantially realistic, although it is not correct to assume that the grain diameter of suspended sediment is equal to that of the bottom sediment.

\section{ADDITIONAL REMARK}

Fig. 17 shows three examples of cumulative plotting of suspended concentration, each representing a different positions of sampling with regard to distance from shoreline and level from the bottom. These data were obtained with an instantaneous horizontal sampler (Fujik1 type) at ojoin, Nilgata (Ref. 17) during December, 1957 to March, 1958. The highest concentration occurs invariably at a bar crest.

The authors have been carrying out a long-term project on the dispersion processes of a free vortex separated from the ripple trough. A minute baffle shaped either triangular or inverted-T is placed on the bottom of a two-dimensional wave flume and the effect of the baffle rise on the dispersion process is studied by visual observation or photograph. F1g. 18 shows an example in which the upper limit $\delta$ of vortex dispersion was compared with that normally observed with natural sand ripples. Observation was facilitated by using aluminium powder or potassium permanganate particles. The relationship between $\frac{\delta}{2}$ and $\frac{u_{m} \eta}{2}$, the Reynolds number with respect to the ripple rise, is fairly consistent and shows that the limiting range of vortex dispersion approaches the water surface as the wave size increases. This suggests that a layer of characteristic turbulence occurs near the bed which is 
SUSPENDED SEDIMENT DUE TO WAVE ACTION

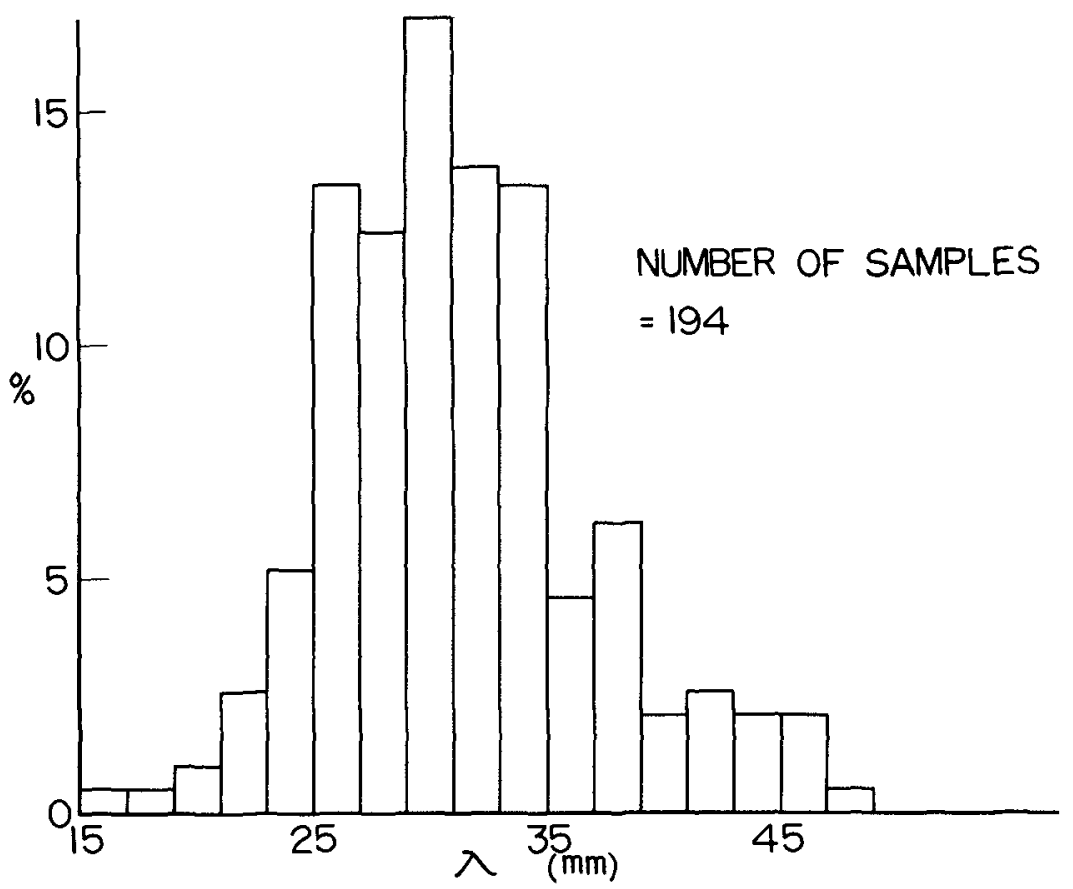

Figure 14. Scatter of ripple pitches found by a single train of waves.

TOKAI COAST

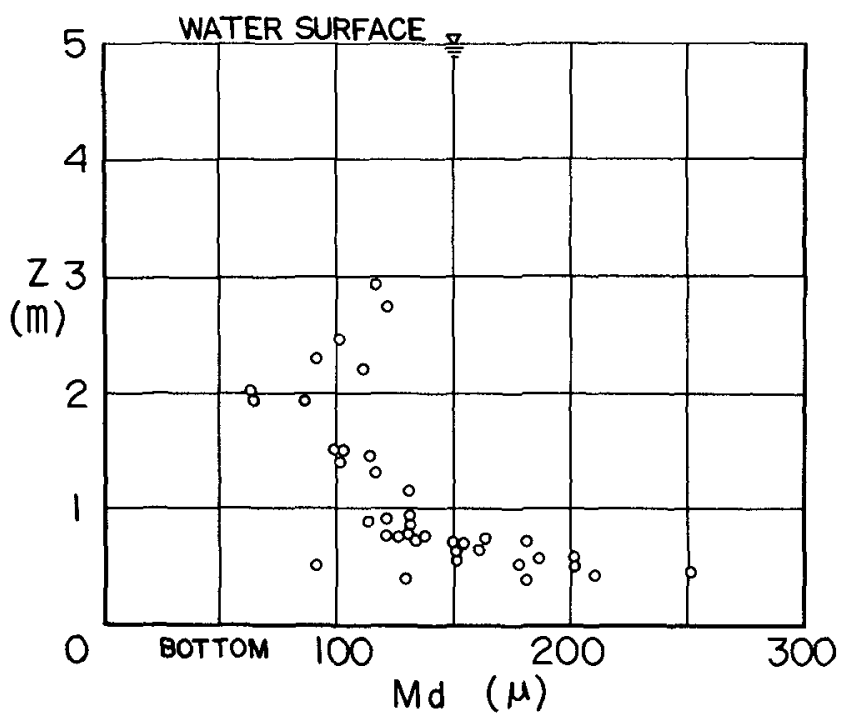

Figure 15. Vertical distribution of medium diameter of suspended sediment (Tokal coast, Japan). 
COASTAL ENGINEERING

NIIGATA COAST

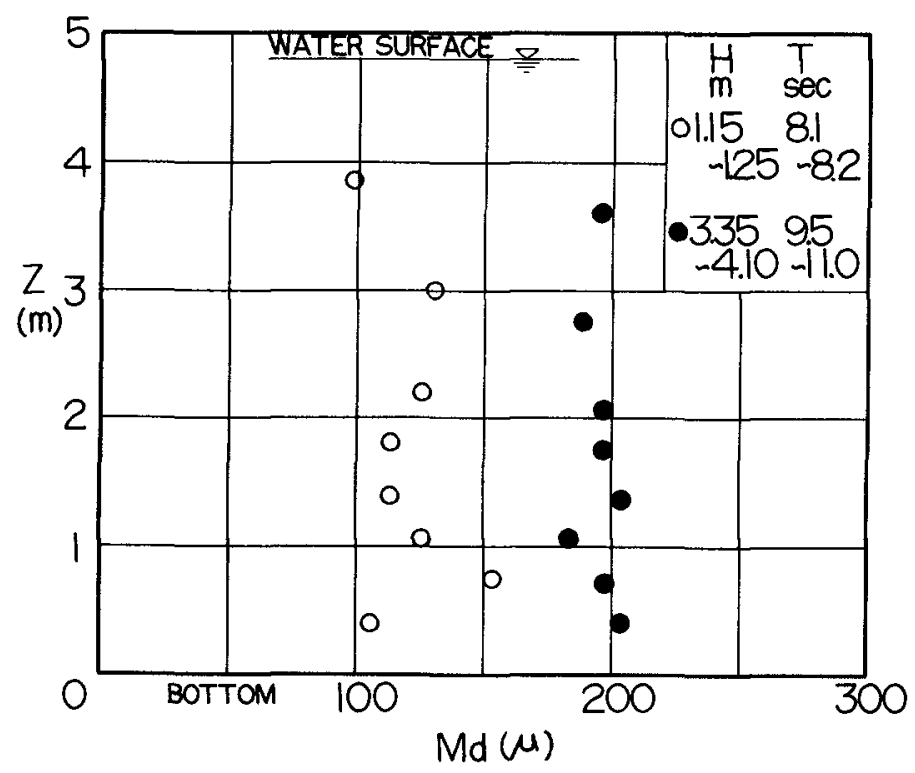

Figure 16. Vertical distribution of medium diameter of suspended sediment (Niigata coast, Japan).

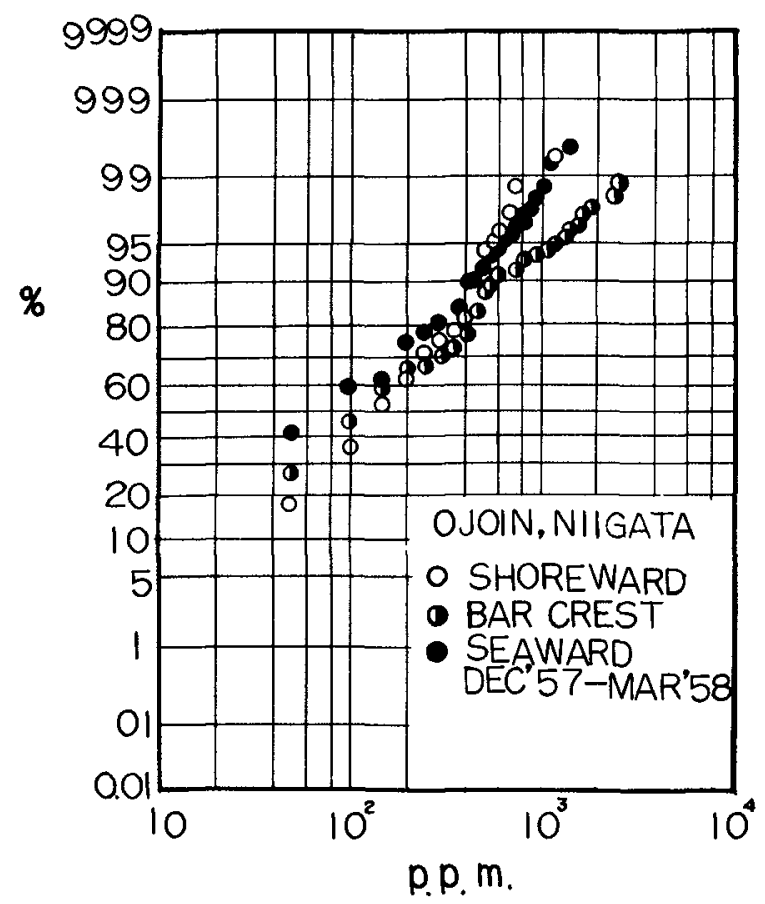

Figure 17. Cumulative occurrence curves of suspended sediment concentration at three different positions (Niigata coast, Japan). 
SUSPENDED SEDIMENT DUE TO WAVE ACTION

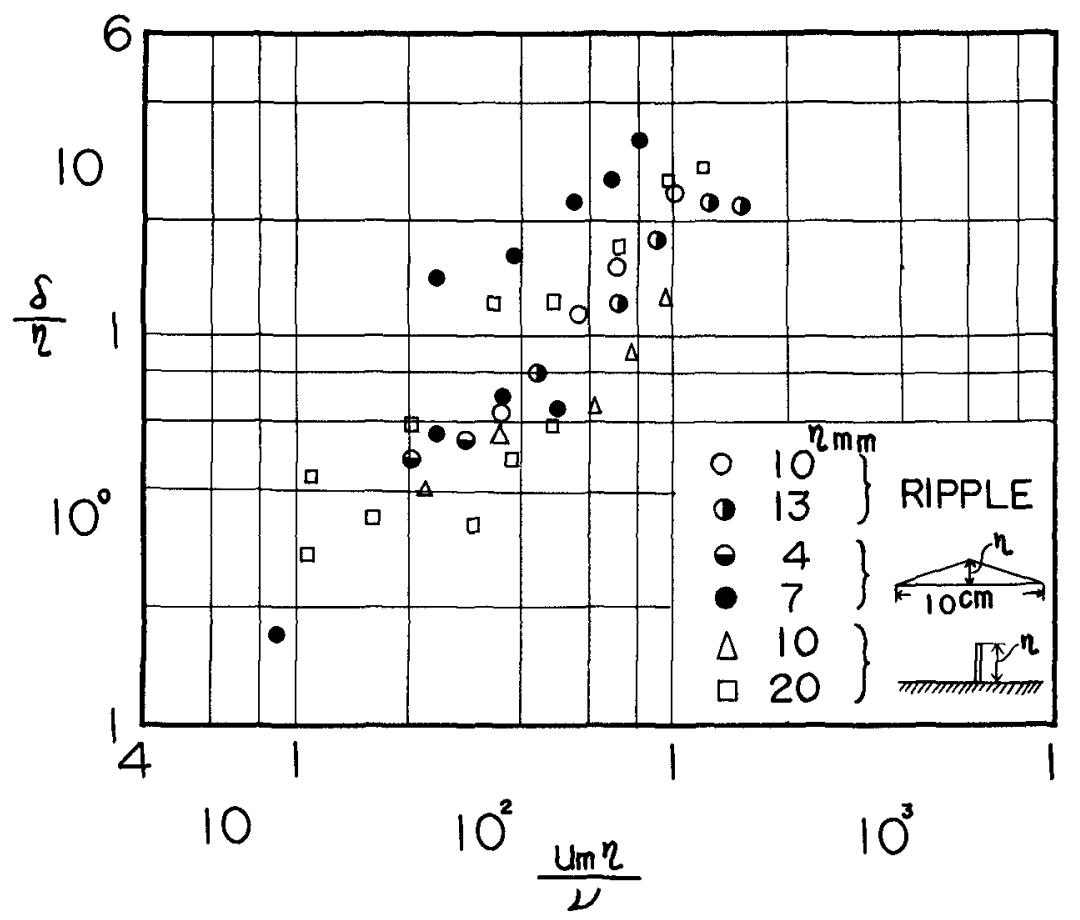

FIgure 18. Relationship between $\delta / \eta$ and $u_{m} \eta / L$.

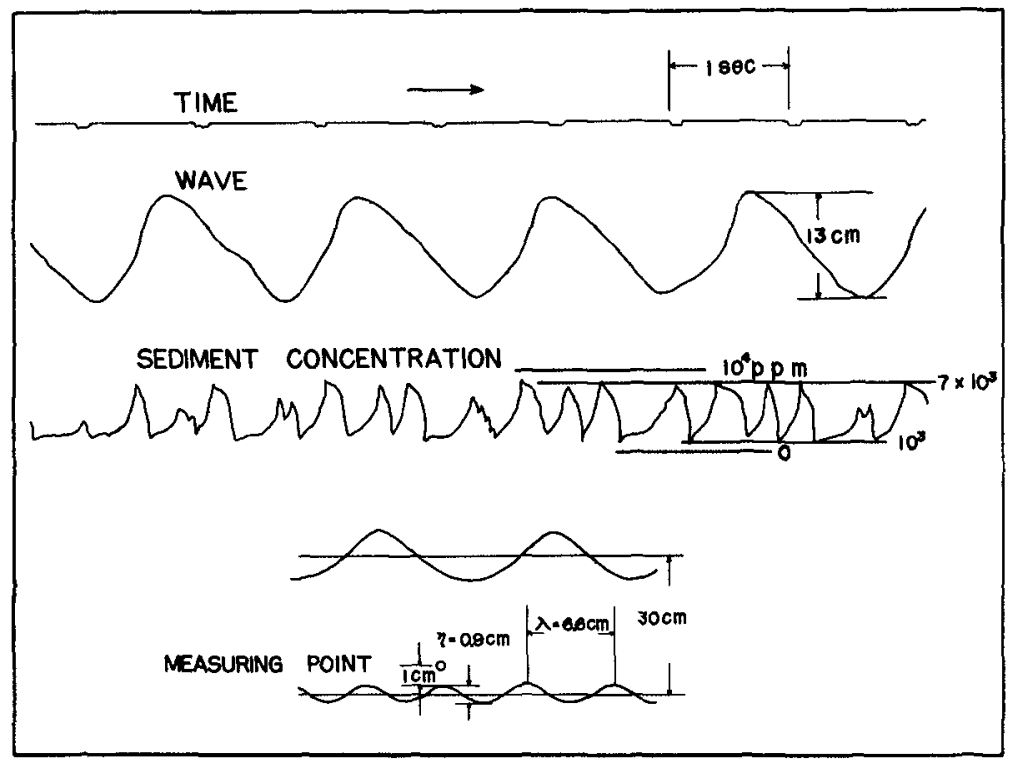

Figure 19. Sample record of suspended sediment concentration varying in time with passing phases of progressive wave (Coastal Engineering Laboratory, University of Tokyo). 


\section{COASTAL ENGINEERING}

acted upon by a relatively small wave, while it grows toward the surface as the wave size increases. Therefore, the assumption of a fully developed turbulence in the body of water which was made in our theoretical treatment should be interpreted to represent rough sea conditions.

Most difficulties in our subject seem to arise from inadequacy of avaliable means of instrumentation. The authors have attempted to develop a device which may enable a continuous recording of suspended concentration which in fact fluctuates following the passage of a wave. Fig. 19 gives one of the preliminary data obtained by using the newly developed model, which works on photoelectric principle with a pick-up unit equipped with a phototransistor element. This instrument is still not fully satisfactory, and must be improved further. According to our measurement, the variation of concentration is much more complicated than has been expected in our theoretical treatment. At any rate the success of recording the actual variation of suspended sediment concentration encourages the authors to advance further with the present study.

\section{CONCLUSIONS}

In this paper the authors summarized the main results of their studies concerning the suspended sediment due to wave action. The data in the field and laboratories were utilized to determine the characteristics of several unknown factors, such as $\alpha$, $\overline{\mathrm{m}}_{\mathrm{a}}$ and $\overline{\mathrm{q}}_{\mathrm{a}}$, which appear in the theory presented here. The agreement between the analytical and measured curves of vertical distribution of suspended sediment concentration is quite satisfactory for the engineering purposes.

In addition to the above, some other data were presented in order to clarify the actual phenomena and to discuss a similarity between prototype and model. In particular, the relationsh $p s, \varphi\left(\frac{\eta}{\lambda}, \frac{\lambda}{d_{0}}, \frac{\eta}{d_{o}} \frac{u_{m} d_{0}}{v}, \frac{u_{0} M d}{y}\right)=0$, as proposed in Figs. 12 and 13, are of prict 1 cal' value to compare the results of field and laboratory studies concerning the bottom sediment movement due to oscillatory wave action. Finally, a continuous recording of the variation of concentration due to progressive wave was shown in Fig. 19, which was made possible by our latest model developed at the Coastal ingineering Laboratory, University of Tokyo. 


\section{SUSPENDED SEDIMENT DUE TO WAVE ACTION}

\section{ACKNOWLEDGEMENTS}

The authors have made extensive reference to a number of existing works concerning the suspended sediment phenomena and the characteristics of sand ripples formed by oscillatory wave action. They should like to express their sincere gratitude and respect to those who have accomplished those initiative studies.

The authors' profound appreciation is due to Dr. Choule Sonu, Coastal Engineering Laboratory, University of Tokyo, who contributed to the operation of field and laboratory works and reviewing the manuscript, and also to the personnel of the same laboratory who assisted in the preparation of figures.

\section{REFERENCES}

1. Fukushima, H. and Y. Mi zoguch1, Drift Sand and Its Measurement, Proc. of 2 nd Conf. on Coastal Engineering in Japan, Nov. 1955. (in Japanese)

2. Fukushima, H., Y. Mizoguchi and M. Kashiwamura, Drift Sand and Its Measurement (2nd Report), Proc. of 4 th Conf. on Coastal Engineering, in Japan, Nov. 1957. (In Japanese)

3. Fukushima, H. and M. Kashiwamura, Drift Sand and Its Measurement ( 3 rd Report), Proc. of 5 th Conf. on Coastal Engineering in Japan, Nov. 1958 (In Japanese)

4. Fukushima, H. and M. Kashiwamura, Drift Sand and Its Measurement (4th Report), Proc. of 6 th Conf. on Coastal Engineering in Japan, Nov. 1959. (In Japanese)

5. Fukushima, H. and Y. Mizoguchi, Field Investigation of Suspended Iittoral Drift, Coastal Eng1neering in Japan, Vol. 1, 1958.

6. Fukushima, H. and M. Kashiwamura, Field Investigation on Suspended Sediment by the Use of Bamboo Samplers, Coastal Engineering in Japan, Vol.2, 1959 .

7. Fukushima, H. and M. Kashiwamura, Some Experiments on Bamboo Samplers, Coastal Engineer1ng in Japan, Vol. 4, 1961 .

8. Kurihara, M., K. Shinohara, T. Tsubaki and M. Yoshioka, Beach Sand Movement due to Waves, Proc. 


\section{COASTAL ENGINEERING}

of 3rd Conf. on Coastal Engineering in Japan, Nov. 1956. (In Japane se)

9. Shinohara, K., T. Tsubaki, M. Yoshotaka and Ch. Agemori, Sand Transport along a Model Sandy Beach by Wave Action, Coastal Engineering in Japan, Vol. 1, 1958.

10. Hom-ma, M., K. Horikawa and C. Sonu, A Study on Beach Sediment at Enoshima and Kamakura, Proc. of 4th Conf. On Coastal Engineering in Japan, Nov. 1957 .

11. Hom-ma, M., K. Horikawa and C. Sonu, A Study on Beach Erosion at the Sheltered Beaches of Katase and Kamakura, Coastal Engineering in Japan, Vol. 3,1960 .

12. Hom-ma, $M$. and $K$. Horikawa, Long shore Current and Sand Drift at Katase East Beach and Kamakura Beach, Investigation Report of Beach Erosion at Katase and Kamakura beaches, Kanagawa Prefecture, Aug. 1958. (In Japanese)

13. Hom-ma, M. (editor), Report of Field Investigations at Tokai-mura (Ist Report), Japan Atomic Generation Company, Aug. 1959. (In Japanese)

14. Horikawa, K. and C. Sonu, Suspended Sediment due to Wave Action, Annual Convention of Japan Society of Civil Engineers, 1960. (In Japanese)

15. Hom-ma, M. and K. Horikawa, Concentration of Suspended Sediment due to Wave Action, Report of Field Investigation at Tokai-mura (2nd Report), Japan Atomic Generation Co., May, 1960. (In Japanese)

16. Nilgata Prefecture, Suspended Sediment at Niigata West Beach, Shin-shi (Collected Data at the Shinano River Construction (ffice), No. 29, Feb. 1958. (In Japanese)

17. Nigata Prefecture, Observation Data on Suspended Sediment, Shin-shi (Collected Data at the Shinano River Construction Office), No. 33-3, 1959. (In Japanese)

18. Watts, G. M., Development and Field Tests of a Sampler for Suspended Sediment in Wave Action, Beach Erosion Board, Tech. Memo. No. 34, March, 1953. 
19. Fairchild, J. C., Development of Suspended Sed1ment Samplers for Laboratory Use under Wave Action, Beach Erosion Board, Bulletin, Vol. 10, No. 1, July, 1956.

20. Fairchild, J. C., Suspended Sediment Sampling in Laboratory Wave Action, Beach Erosion Board, Tech. Memo. No. 115, June, 1959.

21. Bagnold, R. A., Motion of Waves in Shallow Water, Interaction between Waves and Sand Bottoms, Proc. of Royal Society, London, Vol. 187, A, October, 1946.

22. Manohar, M., Mechanics of Bottom Sediment Movement due to Wave Action, Beach Erosion Board, Tech. Memo. No. 75, June, 1955.

23. Scott, T., Sand Movement by Waves, Beach Erosion Board, Tech. Memo. No. 48, Aug. 1954.

24. Inman, D. I., Wave Generated Ripples in Nearshore Sands, Beach Erosion Board, Tech. Memo. No. 100, October, 1957.

25. Goldstein, S., Modern Developments in Fluid Dynamics, Vol. II, 1938.

26. Relf, E. F. and L. F. G. Simmons, The Frequency of the Eddies Generated by the Motion of Circular Cylinders through a Fluid, R. \& M. No. 917, June, 1924, Tech. Rep. of Aeronautical Research Committee, Vol. 2, $1924-5$.

27. Roshko, A., On the Development of Turbulent Wakes from Vortex Streets, National Advisory Committee for Aeronautics, Report 1191, 1955.

28. Vincent, G. E., Contribution to the Study of sediment Transport on a Horizontal Bed due to Wave Action, Proc. of 6 th Conf. on Coastal Engineering, 1958. 